\title{
Pulmonary Venous Doppler Flow Profile before and after Surgical Closure of Atrial Septal Defect in Children
}

\author{
Shrinivas Gadhinglajkar, Rupa Sreedhar, Sabarinath Menon, S Omprakash
}

\begin{abstract}
Objectives: A secundum atrial septal defect is associated with a characteristic pattern of continuous antegrade wave on a pulmonary venous Doppler flow profile. It is replaced with a regular $S$ and $D$ wave after the closure of defect. Although, a few published reports have described these features in adults, there are not enough studies in pediatric patients under anesthesia.
\end{abstract}

Setting: A tertiary referral center.

Participants: A total of 12 children with uncomplicated secundum atrial septal defect participated in the evaluation process.

Interventions: After anesthesia induction, three of them were excluded from detailed pulmonary venous study as they did not satisfy selection criteria. In remaining nine children, the intraoperative pulmonary venous Doppler changes were assessed before and after the defect closure, manually tracing the pulmonary venous waveforms.

Measurements and main results: The antegrade wave was observed in 9/9 patients before surgery that characteristically lacked distinct systolic and diastolic waves, although in seven patients, a larger systolic peak and a smaller diastolic peak were identified.

Normal $S$ and $D$ waves were seen after termination of cardiopulmonary bypass with S/D ratio $>1$ in all cases. The maximum velocity of atrial retrograde wave increased and heart rate decreased significantly after surgery.

Conclusion: The continuous antegrade wave is a consistent pulmonary venous Doppler feature of an uncomplicated secundum ASD in anesthetized children. The Doppler pattern is normalized immediately after termination of cardiopulmonary bypass.

Keywords: Transesophageal echocardiography, Atrial septal defect, Doppler.

How to cite this article: Gadhinglajkar S, Sreedhar R, Menon S, Omprakash S. Pulmonary Venous Doppler Flow Profile before and after Surgical Closure of Atrial Septal Defect in Children. J Perioper Echocardiogr 2013;1(2):42-47.

Source of support: Nil

Conflict of interest: None declared

\section{INTRODUCTION}

A secundum atrial septal defect (ASD) is associated with a characteristic pattern of continuous antegrade wave (CAW) on a pulmonary venous Doppler flow (PVDF) profile. The CAW is replaced with a regular $\mathrm{S}$ wave and a $\mathrm{D}$ wave after the ASD closure.

Even though scanty reports are available in the literature suggesting these changes observed on transesophageal echocardiography (TEE) and transthoracic echocardiography
(TTE), majority of the study subjects included in these studies were adults and the results were evaluated in echo laboratories. ${ }^{1,2}$ Surgical closure of the ASD is routinely performed in pediatric age group. Anesthetic conditions alter pulmonary vascular resistance and left-to-right shunting across the ASD, which may have influence on the Doppler flow pattern of pulmonary veins. It is not clear how early the pulmonary venous flow pattern becomes normal after the surgery. Hence, we evaluated the intraoperative PVDF changes before and after surgical closure of the secundum ASD in children.

\section{MATERIALS AND METHODS}

Intraoperative TEE evaluation was performed in 12 pediatric patients operated for the surgical closure of secundum ASD from June 2012 to March 2013. Informed consent was obtained from the parents of all patients and study clearance was given by the institutional ethics committee. The diagnosis of secundum ASD and normalcy of pulmonary venous drainage was established on TTE in all patients. Four of them had trivial-to-mild mitral regurgitation. According to our institute protocol, TEE probe is inserted only in children weighing more than $5 \mathrm{~kg}$; and hence those weighing less than that were excluded from the observations. Other exclusion criteria included concurrent complex congenital anomalies, sinus venosus $\mathrm{ASD}$, moderate or severe mitral regurgitation, pulmonary hypertension (right ventricular systolic pressure $>30 \mathrm{~mm} \mathrm{Hg}$ ), rhythm other than sinus, pulmonary stenosis and intraoperative inability to interrogate left upper pulmonary vein (LUPV). None of the children were receiving any medication prior to surgery. All of them were premedicated with oral midazolam $0.6 \mathrm{mg} / \mathrm{kg}$ one hour before anesthesia induction. The anesthesia was induced in all cases using sevoflurane in air, supplemented with intravenous injection of fentanyl $5 \mathrm{mcg} / \mathrm{kg}$, midazolam $0.1 \mathrm{mg} / \mathrm{kg}$ and propofol $1 \mathrm{mg} / \mathrm{kg}$. The $\mathrm{FiO}_{2}$ was maintained about 0.25 to 0.3 to achieve $\mathrm{SpO}_{2}>97 \%$. After paralyzing the patient with pancuronium $200 \mathrm{mcg} / \mathrm{kg}$, endotracheal intubation was performed and femoral arterial and right internal jugular venous cannulas were inserted. Routine standard parameters were monitored apart from invasive arterial blood pressure and central venous pressure. Additional fentanyl $10 \mathrm{mcg} / \mathrm{kg}$ and isoflurane 1 to $1.5 \%$ were administered during maintenance of anesthesia. 
A pediatric TEE probe was inserted and cardiac examination was accomplished using a Sonos 7500 machine (Philips Ultrasound, USA) by a consultant anesthesiologists having a 5-year experience in pediatric and adult TEE.

As per the recommendations of American Society of Echocardiography/Society of Cardiovascular Anesthesiologists, a dedicated anesthesiologist was involved in the patient care, when the consultant was busy in performing the TEE examination. Diagnosis of secundum ASD was confirmed on 2D, color flow Doppler (CFD) and pulse wave Doppler (PWD) assessment (Figs 1A and B). The ASD was identified by atrial septal drop-out, right-sided chamber enlargement, paradoxical movement of ventricular septum, and CFD detection of shunt in left-to-right direction. The size of an ASD was measured at the width of color flow across the ASD. Pulmonary artery systolic pressure was estimated from a tricuspid regurgitant jet. Presence of an additional ASD or a patent foramen ovale was noted. Heart was inspected to rule out coexistence of any other congenital anomalies and pulmonary stenosis. Although, all pulmonary veins were interrogated on TEE, the PVDF profiles were recorded from the LUPV. TEE probe was
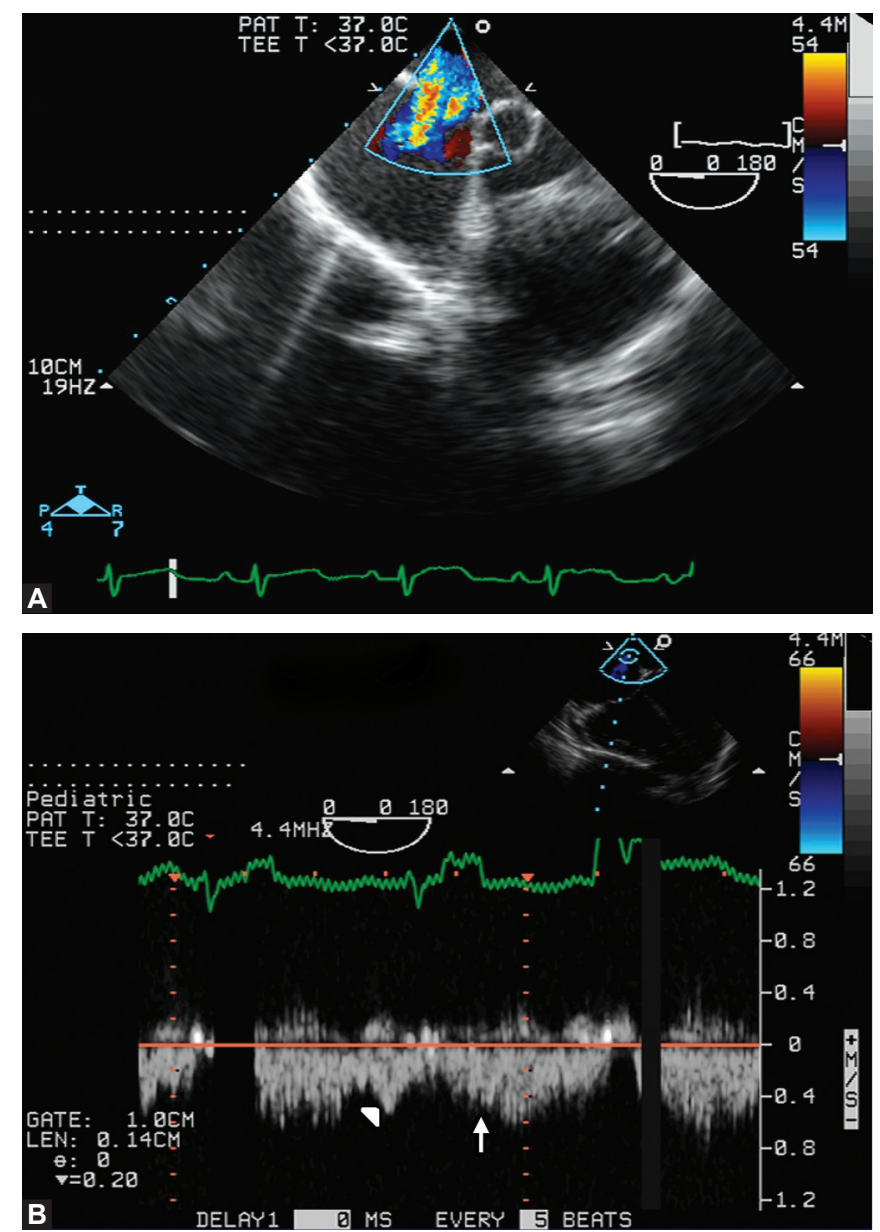

Figs 1A and B: (A) Color flow across the ASD during systole, $(B)$ pulse-wave Doppler interrogation at ASD is revealing flow through the ASD in diastole (arrow head) and systole (arrow) (ASD: Atrial septal defect) slightly turned anticlockwise from midesophageal fivechamber view to obtain a clear view of the LUPV as it empties into left atrium (LA). The sector angle was adjusted between 0 and $30^{\circ}$ to maintain the pulmonary venous flow parallel to the ultrasound beam. A sample volume was placed $1 \mathrm{~cm}$ from pulmonary vein from its junction with LA. By adjusting gain settings, optimum quality of PWD signals was obtained. Signals having greatest amplitude were selected for measurements. Baseline Doppler profile of pulmonary veins was recorded before sternotomy. Left lower pulmonary vein was visualized by further increasing the sector angle; and by leftward rotation and deeper insertion of the probe. The right-sided pulmonary veins were inspected at $0^{\circ}$ and also at extended bicaval view at 90 to $120^{\circ}$ with rightward rotation of the probe, where the vein is seen adjacent to the right pulmonary artery. A pericardial patch was harvested after sternotomy for the closure of ASD.

After administration of 400 units $/ \mathrm{kg}$ of heparin to achieve activated clotting time $>480$ seconds, cardiopulmonary bypass (CPB) was established with single-stage cannulae placed in superior and inferior vena cavae. The aorta was cross-clamped and antegrade cold blood cardioplegia was infused into the aortic root to achieve cardiac electromechanical quiescence. After surgical closure of the ASD, the heart was de-aired via aortic root vent and the aorta was de-clamped. None of the patients required inotropic support while weaning them from CPB. The PVDF data was recorded in the post-CPB period in the same manner as in pre-CPB period. All children were electively ventilated in the postoperative period and weaned off ventilator within 6 to 8 hours. All of them had an uneventful postoperative recovery.

The Doppler profile of pulmonary veins was examined off-line and waveforms were identified and traced manually in concurrence with ECG as described by Tanaka $\mathrm{K}$ et $\mathrm{al}^{3}$ and Intsugi $\mathrm{M}$ et al. ${ }^{4}$ The ECG periods of systole, early-diastole and atrial contraction phase corresponded with summit of the $\mathrm{R}$-wave to end of T-wave, end of T-wave to end of P-wave and end of $\mathrm{P}$-wave to summit of $\mathrm{R}$-wave respectively. CAW was identified in the pre-CPB period as a continuous wave in both systole and early diastole. Two different patterns of CAW, either a single systolic peak (S-peak) or an S-peak and a diastolic peak (D-peak) were noted (Figs 2A and B). The CAW was traced manually and its mean velocity and velocity-time integral (VTI) were measured. Similarly, the peak velocity, mean velocity and VTI of the atrial reversal wave (ARW) were assessed after tracing the wave manually.

After surgery, peak velocity, mean velocity and VTI of the S-wave, D-wave and ARW were measured (Fig. 3). Three consecutive beats were traced and results were averaged in each patient. The hemodynamic data was collected from the monitoring trends. 

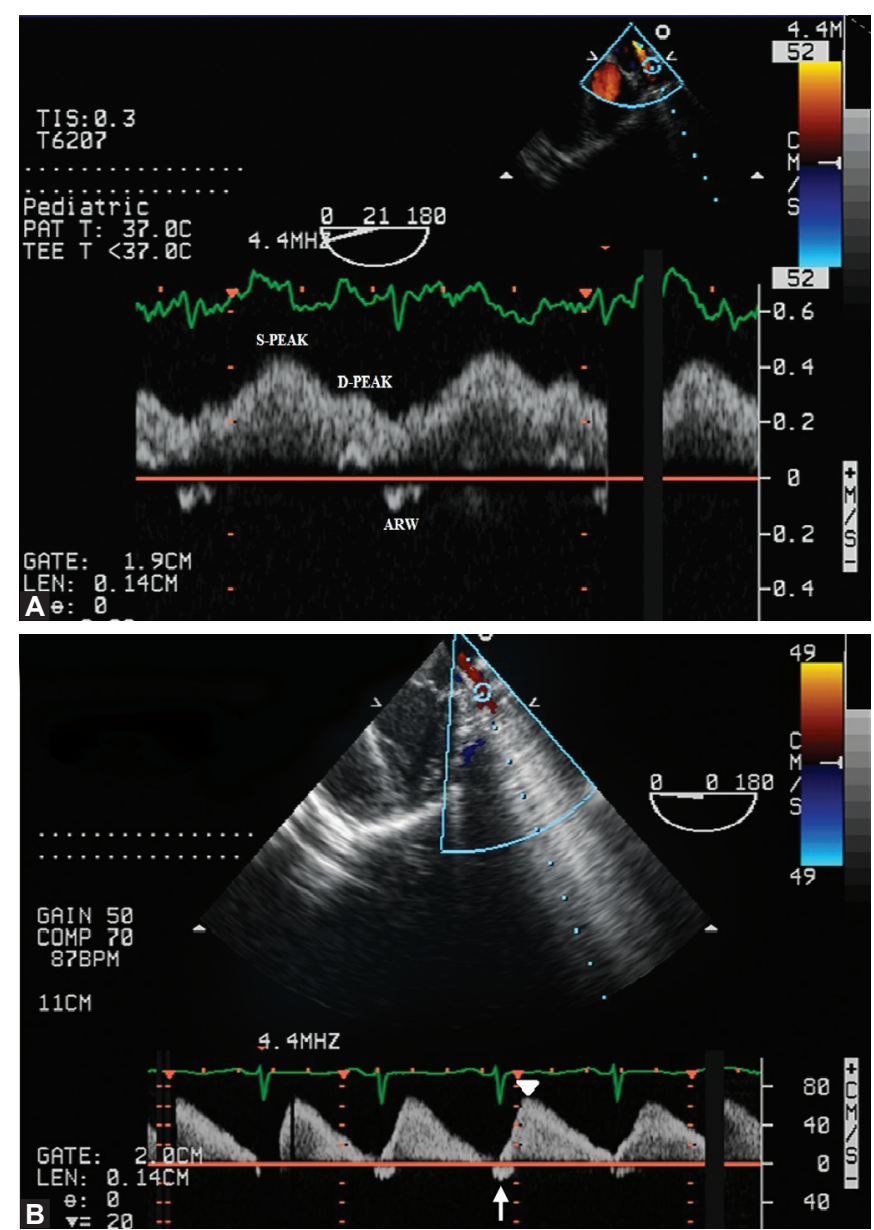

Figs 2A and B: Left upper pulmonary vein Doppler interrogation is revealing two patterns of CAW in the pre-CPB period. Characteristically, the systolic and diastolic waves are not separable in the CAW. The ARW is also visible: (A) CAW with a S-peak in late systole and a D-peak in early-diastole, (B) CAW having only one systolic peak (arrow head). The arrow is pointing the ARW (CAW: Continuous antegrade wave; CPB: Cardiopulmonary bypass; ARW: Atrial reversal wave)

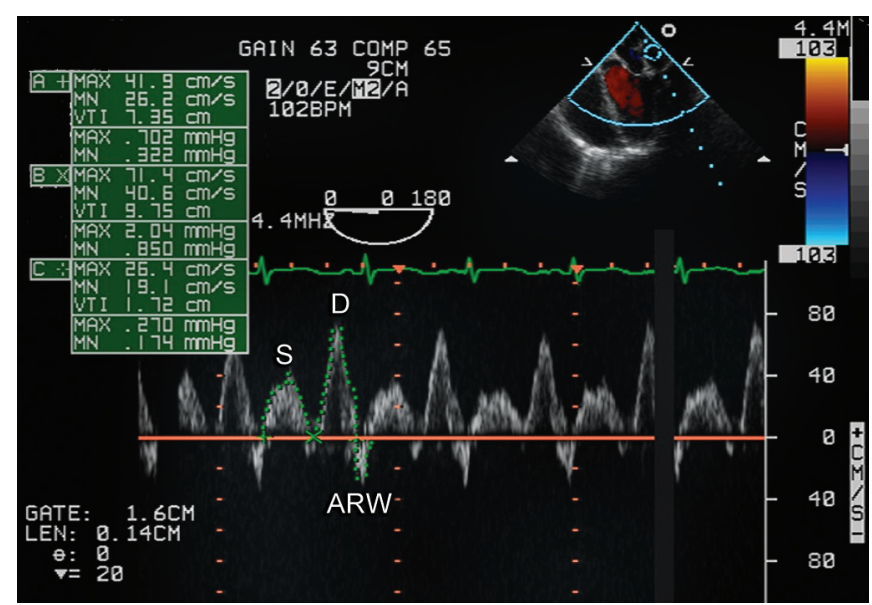

Fig. 3: Pulmonary venous Doppler flow profile in the post-CPB period is displaying distinctly separable S-wave, D-wave and a prominent ARW. The SD ratio is $>1$

The data was analyzed statistically using student t-test for comparison between group means. The difference between groups was considered significant when the twotailed p-value was less than 0.05 .

\section{RESULTS}

Total 12-children were selected for the assessment of intraoperative TEE changes before and after the surgical closure of ASD. One of them was found to have a mild pulmonary stenosis with a systolic gradient of $25 \mathrm{~mm} \mathrm{Hg}$ across the pulmonary valve on intraoperative TEE. Another child had a TEE finding of an anomalous drainage of right upper pulmonary vein into superior vena cava - right atrium junction. The mitral regurgitation was found to be severe in a child. All three of them were excluded from the subjects considered for TEE evaluation during surgery. The mean and standard deviations of age and weight of remaining nine patients ( 5 females and 4 males) who satisfied the inclusion criteria are listed in Table 1. One of them had an additional patent foramen ovale, which was seen on color flow Doppler. The age of oldest child among the patients was 8 years. The size of ASD ranged from 7.8 to $25.3 \mathrm{~mm}$ (mean SD: 13.54 $\pm 5.68 \mathrm{~mm}$ ). All of them had left-to-right shunting across the ASD, which was verified on CFD and PWD. The heart rate reduced in seven patients after ASD closure, which was statistically significant ( $\mathrm{p}$-value $<0.05$ ).

\begin{tabular}{lcc}
\multicolumn{3}{c}{ Table 1: Demographic details and size of ASD $(\mathrm{n}=9)$} \\
\hline & Range (median) & Mean \pm SD \\
\hline Age (months) & $36-96(42)$ & $56.1 \pm 22.32$ \\
Weight $(\mathrm{kg})$ & $9.0-24.0(13)$ & $14.1 \pm 5.2$ \\
Size of ASD $(\mathrm{mm})$ & $7.8-25.3(11.9)$ & $13.54 \pm 5.68$ \\
\hline
\end{tabular}

SD: Standard deviation; ASD: Atrial septal defect

All pulmonary venous Doppler measurements, summarized in Table 2, were performed when the heart was beating in sinus rhythm. The CAW was observed in 9/9 patients in the pre-CPB period that characteristically lacked separable systolic and diastolic waves, although in seven patients, an S-peak and a D-peak were identified. The maximum velocity of S-peak was larger than that of D-peak in all seven cases, (S-peak/D-peak ratio mean \pm SD: 1.24 \pm 0.11 ). In remaining two of the patients, the CAW had a single peak during systole. Even though separate S-peak and D-peak were seen in the CAW, we measured the velocities and VTI of the CAW as a whole since the $\mathrm{S}$ and $\mathrm{D}$ waves were not distinct. No direct correlation was noticed between the size of ASD and velocities or VTI of CAW. A small ARW was present in all subjects in the pre-CPB period. The CAW was replaced with normal S-wave and D-wave in the post$\mathrm{CPB}$ period. The $\mathrm{D}$-wave was prominent in all children, with its maximum velocity higher than the maximum velocity of S-wave $\left(\mathrm{S}_{\mathrm{MAX}} / \mathrm{D}_{\mathrm{MAX}}\right.$ ratio, mean $\pm \mathrm{SD}$ : $\left.0.65 \pm 0.13\right)$. The maximum velocity of RAW was significantly enhanced after surgical closure of ASD compared to pre-CPB period. Although, there was overall increase in the mean velocity and VTI of ARW after surgery, it was statistically not significant. 
Pulmonary Venous Doppler Flow Profile before and after Surgical Closure of Atrial Septal Defect in Children

\begin{tabular}{|c|c|c|c|c|c|c|}
\hline \multirow[t]{2}{*}{ Parameters } & \multirow[t]{2}{*}{$n$} & \multicolumn{2}{|c|}{ Pre-ASD closure } & \multicolumn{2}{|c|}{ Post-ASD closure } & \multirow[t]{2}{*}{$p$-value } \\
\hline & & Range (median) & Mean $\pm S D$ & Range (median) & Mean $\pm S D$ & \\
\hline HR (bpm) & 9 & $80-118(105)$ & $101.1 \pm 12.9$ & $79-108(94)$ & $95.6 \pm 9.2$ & $0.03016^{*}$ \\
\hline S-peak (cm/sec) & 7 & $35.9-55.9(48.5)$ & $45.6 \pm 6.9$ & - & - & - \\
\hline D-peak (cm/sec) & 7 & $31.6-41.7(35.8)$ & $36.9 \pm 4.6$ & - & - & - \\
\hline S-peak/D-Peak ratio & 7 & $1.10-1.42(1.35)$ & $1.24 \pm 0.11$ & - & - & - \\
\hline $\mathrm{ARW}_{\text {MAX }}(\mathrm{cm} / \mathrm{sec})$ & 9 & $12.5-17.7(14)$ & $14.4 \pm 1.6$ & $13.2-26.4(16.2)$ & $17.5 \pm 3.7$ & $0.04559^{*}$ \\
\hline $\mathrm{ARW}_{\text {MEAN }}(\mathrm{cm} / \mathrm{sec})$ & 9 & $9.1-15.6(12.4)$ & $12.2 \pm 2.3$ & $11.0-19.1(12.5)$ & $13.1 \pm 2.7$ & 0.34127 \\
\hline $\mathrm{ARW}_{\mathrm{VTI}}(\mathrm{cm})$ & 9 & $0.7-1.8(1.1)$ & $1.1 \pm 0.3$ & $1.0-1.7(1.1)$ & $1.2 \pm 0.3$ & 0.30459 \\
\hline $\mathrm{S}_{\mathrm{MAX}}(\mathrm{cm} / \mathrm{sec})$ & 9 & - & - & $25.6-41.9(32.4)$ & $32.6 \pm 5.2$ & - \\
\hline $\mathrm{S}_{\text {MEAN }}(\mathrm{cm} / \mathrm{sec})$ & 9 & - & - & $14.3-27.6(22.5)$ & $21.8 \pm 4.5$ & - \\
\hline $\mathrm{S}_{\mathrm{VTI}}(\mathrm{cm})$ & 9 & - & - & $4.4-8.4(5.9)$ & $6.2 \pm 1.3$ & - \\
\hline $\mathrm{D}_{\operatorname{MAX}}(\mathrm{cm} / \mathrm{sec})$ & 9 & - & - & 38.3-71.4 (51.2) & $52.9 \pm 9.9$ & - \\
\hline $\mathrm{D}_{\text {MEAN }}(\mathrm{cm} / \mathrm{sec})$ & 9 & - & - & $22.5-40.6(32.2)$ & $31.9 \pm 5.5$ & - \\
\hline $\mathrm{D}_{\mathrm{VTI}}(\mathrm{cm})$ & 9 & - & - & $5.7-12.1(9.8)$ & $9.4 \pm 1.8$ & - \\
\hline$S_{\text {MAX }} / D_{\text {MAX }}$ ratio & 9 & - & - & $0.51-0.85(0.63)$ & $0.65 \pm 0.13$ & \\
\hline
\end{tabular}

HR: Heart rate; n: number of patients; CAW: Continuous antegrade wave; ARW: Atrial reversal wave; MAX: Maximum velocity; MEAN: Mean velocity; VTI: Velocity-time integral; SD: Standard deviation; * Indicates statistical significance ( $\mathrm{p}$-value $<0.05$ using 2 -tailed student t-test)

\section{DISCUSSION}

An important characteristic feature of an uncomplicated secundum ASD is the presence of a CAW on Doppler interrogation of pulmonary veins, which is replaced with regular S-wave and D-wave after the surgical closure of ASD. It is also accompanied with an increase in the peak velocity of ARW. These changes were demonstrated on $\mathrm{TEE}^{1}$ and $\mathrm{TTE}^{2}$ in previous published reports involving mainly adult patients. Schroh et al ${ }^{5}$ have described a similar pattern of pulmonary venous flow on transthoracic Doppler examination in pediatric patients. Our observations on PVDF changes in children under anesthesia are consistent with the observations from previously described studies. We selected the pediatric patient because the ASD closures are routinely performed in this age group. Assessment performed in an echolaboratory or during a postoperative review might differ from the intraoperative assessment under anesthesia. Theoretically, the anesthetic agents, oxygen and positive pressure ventilation may affect the pattern of the CAW by modulating pulmonary vascular resistance and pulmonary blood flow. However, we noticed a similar pattern of CAW in pre-CPB period and a normalized configuration of S-wave, D-wave and ARW immediately after termination of the CPB.

Apart from the secundum ASD, pulmonary venous stenosis $^{6}$ is another condition in which a CAW may be detected on a PVDF examination. It is identified by the presence of a turbulent jet originating from a pulmonary vein that is accompanied with a continuous high peak velocity flow $(>2 \mathrm{~m} / \mathrm{sec})$ on PWD. ${ }^{6}$ Excluding this rare condition, presence of a CAW is almost a diagnostic feature of a secundum ASD with left-to-right shunt. Normalization of the pulmonary venous waveforms immediately after the ASD closure suggests that the pre-CPB continuous flow in pulmonary veins is attributed to a left-to-right shunting. In fact, we suggest that finding this peculiar CAW pattern on PVDF should alert an echocardiographer of the possibility of an underlying undiagnosed secundum ASD, which is concomitantly discovered in many congenital cardiac conditions. Pulmonary venous catheterization studies in patients with ASDs have depicted two different types of antegrade waveforms. ${ }^{7,8}$ One was a waveform of two peaks with the first peak in late systole and the second peak in early diastole. The other was a waveform of one peak with a summit near the end of systole. We observed both patterns on TEE in our patients. The pattern of a singlepeak was observed in two patients who had a large size of ASD (19.3 and $25.3 \mathrm{~mm}$ ) and a pattern of double-peak was recorded in remaining seven patients. Schroh et $\mathrm{al}^{5}$ stated that the maximum velocity of the systolic portion of a CAW always exceeds that of the diastolic portion. The mean ratio of S-peak to D-peak of the CAW in our patients was 1.24.

Mechanism of generation of a continuous wave may be explained on the basis of left-to-right shunting across the $\mathrm{ASD}$, which is a low resistance pathway available for the left atrium (LA) to decompress during various phases of cardiac cycle. Pulsed Doppler signals in the ASD and pulmonary vein indicate that the LA decompression occurs continuously both during systole and diastole. In normal circumstances, 
the pulmonary venous flow is accelerated during ventricular systole and diastole due to a phasic reduction in the left atrial pressure. This phasic influence of ventricles on left atrial pressure is retained in patients with ASD to some extent which contributes to the formation of systolic and diastolic peaks on a CAW. Schroh et al have demonstrated that tricuspid annular descent is increased in the presence of right ventricular volume overloading, which enhances the right ventricular systolic function. The right ventricular systole probably exerts a suction effect on the right atrium, which favors forward propulsion of blood across the vena cavae and the ASD that results in a large S-peak on the continuous waveform. Since, the suction effect of RV is less effective in diastole compared to systole in these patients, the velocity of D-peak remains less than the velocity of S-peak. Schrof et al did not find any gross difference in left ventricular systolic and diastolic function in patients with ASD compared to that in the healthy children. Hence, they concluded that the pulmonary venous flow is less affected by the phasic activity of left ventricle compared to the right ventricle. We observed a single systolic-peak on the continuous wave in patients with a large ASD, which we attribute to a strong right ventricular systolic contraction preceding a blunted diastolic effect of both ventricles on the left atrial pressure.

Normal pulmonary venous Doppler waveforms consist of an S-wave, D-wave and a wave of atrial reversal. The S-wave (sometimes 2 components: S1 and S2) represents flow into the LA during ventricular systole and is due to atrial relaxation combined with suction effect of the base to apex movement of the heart as it contracts and twists. The D-wave corresponds with fall in the left atrial pressure during the early diastolic period that promotes forward flow in the pulmonary veins. In pediatric population, the D-wave is usually larger than the $\mathrm{S}$-wave. The $\mathrm{S} / \mathrm{D}$ ratio increases as child grows to adulthood. There is a brief reversal of pulmonary venous flow during late-diastole associated with atrial systole generating ARW. ${ }^{9}$ The height of ARW was low before commencement of the $\mathrm{CPB}$, probably because the LA was emptying actively into the right atrium and left ventricle during the atrial contraction phase that allowed only a small pressure wave to travel into the pulmonary vein in a retrograde fashion. After ASD closure, the amplitude of ARW increased significantly, suggesting a mild increase in the LA pressure during atrial contraction.

The LUPV is suitable for Doppler interrogation, as it can be easily visualized on TEE and aligned parallel to the ultrasound probe. As the normal pulmonary veins lack venous valves, pressure changes in the LA are reflected accurately on the pulmonary venous Doppler waveforms. Even though, we interrogated the LUPV, we confirmed that other pulmonary veins have had a normal drainage into the LA. Usually, the PVDF pattern remains similar in all pulmonary veins ${ }^{1}$. It is not influenced by respiration and primarily determined by the performance of the left side of heart. TEE evaluation of pulmonary venous Doppler pattern in patients with the secundum ASD is suited in a cardiac surgical set up as TEE is widely available. TEE is highly sensitive in detecting an ASD, because the proximity and orientation of the atrial septum relative to the esophagus permits the entire structure to be adequately visualized in virtually every patient. ${ }^{10,11}$ We are aware that the patient cohort was small in size and larger studies in future would be necessary to project a better level of evidence. We did not correlate alterations in PVDF profile to the changes in cardiac output or left-to-right shunting, because quantifying cardiac output was not the objective. The PVDF changes were assessed in patients with an uncomplicated secundum ASD. These observations probably would have been different in the presence of severe pulmonary hypertension and pulmonary stenosis. Although, we measured the pulmonary venous VTIs, we did not calculate flow volume, as measuring diameter of pulmonary veins would have been difficult in an intraoperative scenario. We did not correlate changes in pulmonary venous Doppler with transmitral diastolic flow pattern because LV diastolic dysfunction was very less likely to exist in this age group.

\section{SUMMARY}

The continuous antegrade wave is a consistent pulmonary venous Doppler feature of an uncomplicated secundum ASD in anesthetized children. The Doppler pattern is normalized immediately after termination of cardiopulmonary bypass.

\section{REFERENCES}

1. Saric M, Applebaum RM, Phoon CK, Katz ES, Goldstein SA, Tunick PA, Kronzon I. J Am Soc Echocardiogr 2001;14:386-390.

2. Chockalingam A, Dass S, Alagesan R, Muthukumar D, Rajasekar MA, Subramaniam T, Jaganathan V, Elangovan S. Role of transthoracic Doppler pulmonary venous flow pattern in large atrial septal defects. Echocardiography 2005;22:9-13.

3. Tanaka K, Kitahata H, Kawahito S, Nozaki J, Tomiyama Y, Oshita S. Phenylephrine increases pulmonary blood flow in children with tetralogy of fallot. Can J Anaesth 2003;50:926-929.

4. Inatsugi M, Tanaka K, Kitahata H, Nozaki J, Kawahito S, Oshita S. Minute distance obtained from pulmonary venous flow velocity using transesophageal pulsed Doppler echocardiography is related to cardiac output during cardiovascular surgery. J Med Invest 2005;52:178-185.

5. Schroh AM, Laghezza LB, Domínguez PJ, Brandán V, Nento DE, Alvarez E, Farinelli CE. Pattern of pulmonary venous flow in patients with ostium secundum atrial septal defect. Eur J Echocardiogr 2009;10:244-249. 
6. Ha JW, Chung N, Yoon J, Jang Y, Kim BO, Cho SY, Cho BK. Pulsed wave and color Doppler echocardiography and cardiac catheterization findings in bilateral pulmonary vein stenosis. J Am Soc Echocardiogr 1998;11:393-396.

7. Takaya T, Arakawa M, Tanaka T, Goto M, Yamaguchi M, Nagano T, Miyamoto H, Hirakawa S. Pulmonary vein blood flow velocity waveform with special reference to pulmonary 'systolic runoff' in patients with atrial septal defect. Jpn Circ J 1986;50:405-415.

8. Lam YY, Fang F, Yip GW, Li ZA, Yang Y, Yu CM. New pulmonary vein Doppler echocardiographic index predicts significant interatrial shunting in secundum atrial septal defect. Int J Cardiol 2012 Sep 20;160(1):59-65.

9. Sidebotham D, Hussey M. Left ventricular diastolic dysfunction. In: Sidebotham D, Merry A, Legget M, editors. Practical perioperative transesophageal echocardiography. London, Butterworth Heinemann 2003:117-129.

10. Dinardo JA, Echocardiographic evaluation of intracardiac masses and septal defects. In: Konstatd SN, Shernan SK, Oka Y, editors. Clinical transesophageal echocardiography. Lippincott Williams and Wilkins, Philadelphia 2003:191-202.

11. Adult congenital heart diseases. In: Oxorn DC, Otto CM, editors. Atlas of intraoperative transesophageal echocardiography. Saunders Elsevier, Philadelphia 2007:238-283.

\section{ABOUT THE AUTHORS}

\section{Shrinivas Gadhinglajkar (Corresponding Author)}

Professor, Department of Anesthesia, Sree Chitra Tirunal Institute for Medical Sciences and Technology, Thiruvananthapuram Kerala-695011,India, Phone: 0091 4712443152,Fax: 009104712446433 e-mail: shri@sctimst.ac.in

\section{Rupa Sreedhar}

Professor, Department of Anesthesia, Sree Chitra Tirunal Institute for Medical Sciences and Technology, Thiruvananthapuram, Kerala, India

\section{Sabarinath Menon}

Assistant Professor, Department of CVTS, Sree Chitra Tirunal Institute for Medical Sciences and Technology, Thiruvananthapuram, Kerala India

\section{S Omprakash}

Senior Resident, Department of Cardiothoracic and Vascular Anesthesia, Sree Chitra Tirunal Institute for Medical Sciences and Technology, Thiruvananthapuram, Kerala, India 